\title{
Treatment Standards and Individualized Therapy of Myasthenia
} Gravis

\section{(우 (1) $(8) \Theta$}

Authors

Peter Paul Urban ${ }^{1}$, Christian Jacobi ${ }^{2}$, Sebastian Jander ${ }^{3}$

\begin{abstract}
A wide range of established treatment options is currently available for myasthenia gravis. These include cholinesterase inhibitors for symptomatic treatment and a broad spectrum of immunosuppressive, immunomodulating or cell-depleting options to modify the underlying immunological process. Appropriate use allows the great majority of patients to lead a normal life. Specialized centers integrating outpatient and in-hospital resources as well as interdisciplinary competences offer important advantages for optimum individualized therapy.
\end{abstract}

\author{
Bibliography \\ ISSN 2511-1795 \\ Correspondence \\ Prof. Dr. med. Sebastian Jander, \\ Klinik für Neurologie, \\ Universitätsklinikum Düsseldorf, \\ Moorenstr. 5 \\ 40225 Düsseldorf \\ Germany \\ jander@uni-duesseldorf.de
}

DOI https://doi.org/10.1055/s-0043-124983

Neurology International Open 2018; 2: E84-E92

(c) Georg Thieme Verlag KG Stuttgart · New York

\section{Introduction}

Myasthenia gravis is a well-treatable disease. Under optimal standard therapy, approximately 85-90\% of all patients achieve good treatment results with extensive remission of myasthenic symptoms and recovery of everyday life skills [1]. This review examines the established therapeutic standards that include both symptomatic therapy with acetylcholinesterase inhibitors as well as a wide range of immunologically-based therapies. Additional articles in a special issue of Aktuelle Neurologie deal with the limitations and possibilities of drug therapy during pregnancy and lactation as well as further therapeutic options for therapy-resistant myasthenia as well as escalation therapy for myasthenic crisis.

\section{Symptomatic Therapy with \\ Acetylcholinesterase Inhibitors}

Early investigations of the use of acetylcholinesterase inhibitors (AchEI) to treat myasthenia gravis (MG) were performed in the 1930s. In 1931 prostigmine (neostigmine) was first synthesized and appeared to be a promising drug [2]. Lazar Remen, while working at the Münster University Hospital, was probably the first physician to treat a patient with myasthenia gravis using prostigmine, which resulted in an improvement of myasthenic symptoms lasting one hour [3]. The English physician Mary Walker recognized the similarities between curare poisoning and myasthenia gravis. She then treated a myasthenia gravis patient with physiostigmine and subsequently with prostigmine, particularly to reduce CNS side effects. The patient thus treated exhibited an impressive improvement of clinical symptoms. In an article published in 1934, the effects are also shown photographically with a clear improvement in the right-pronounced ptosis and ocular deviation [4]. Further AchEI variants were synthesized in the following decades. In particular, the substances still used today include pyridostigmine bromide, ambenonium chloride, distigmine bromide and edrophonium chloride. 


\section{Action Mechanism}

Acetylcholine is degraded by acetylcholinesterase (AchE) via hydrolysis. Inhibition of acetylcholine by AchE inhibitors is caused by competitive binding to the enzyme, which leads to significantly slower hydrolysis of AchEl compared to acetylcholine, and thus to reversible blockage of the enzyme. The degradation of acetylcholine is consecutively reduced, and there is an increase in acetylcholine in the synaptic cleft, which in turn leads to an improvement in the neuromuscular signal transmission. The duration of the effect depends on the stability of the temporary binding (AchE-AchEI) [5]. The AchE inhibitors used as the hydrophilic quaternary ammonium compounds do not cross the blood-brain barrier at the usual therapeutic dose, or only in very low concentrations, so that central nervous system side effects of these substances usually only occur in intoxication.

\section{Value in Therapy of Myasthenia}

The effect of AchE inhibitors is clinically undisputed, but is based only on electrophysiological studies and not on controlled clinical studies [6-9]. A recent meta-analysis revealed only a published abstract of a controlled randomized study on the intranasal administration of neostigmine in 10 patients [8]. However, future placebo-controlled randomized trials are unlikely to be expected due to the efficacy of AchE inhibitors in routine treatment.

\section{Information Regarding Available Individual Substances}

Pyridostigmine is the most commonly used AchE inhibitor with fewer muscarinic side effects compared to neostigmine. Generally, dosage is between 60 to $90 \mathrm{mg}$ tablets per single dose every 4 to $6 \mathrm{~h}$, although it is useful to titrate pyridostigmine (for example, an initial single dose of $30 \mathrm{mg}$, sometimes 3 to $4 \times 20 \mathrm{mg}$ for purely ocular MG, in less common cases $3 \times 10 \mathrm{mg} / \mathrm{d}$ is sufficient). If there is additional immunosuppressive therapy, the dose may be reduced in many cases. Conversely, extraordinary physical exertion, infections or MG-aggravating comedications may also necessitate a dose increase. Clinical effectiveness generally starts after approx. 15 to $30 \mathrm{~min}$ and continues for 2 to $4 \mathrm{~h}$. Daily doses of less than $300 \mathrm{mg}$ usually do not lead to overdose symptoms, dosages above $600 \mathrm{mg} / \mathrm{d}$ should generally be avoided due to the risk of cholinergic crisis. Individual patients, especially those with a still present decrement in $3 \mathrm{~Hz}$ stimulation (evidence of a so-called "cholinergic reserve"), may benefit from higher doses without side effects. It is possible to pestle pyridostigmine bromide and then dissolve it in water, thus making administration possible in the case of dysphagia, or via a nasogastric tube.

Additionally an extended-release form of pyridostigmine is available (dosage: $90-180 \mathrm{mg}$ ). The effect takes somewhat longer than the non-extended-release form (after approx. $60 \mathrm{~min}$ ); and the maximum effectiveness occurs after 6 to $10 \mathrm{~h}$. Extended-release pyridostigmine bromide may be helpful in case of fluctuations of effect or patients with nocturnal or early morning symptoms.

Pyridostigmine may also be administered parenterally (IM or IV); it should be noted that the dosage should be reduced by a factor of 30 ; i. e. $1 \mathrm{mg} \mathrm{IV}$ is equivalent to an oral dose of $30 \mathrm{mg}$. Gen- erally $0.5-2 \mathrm{mg} / \mathrm{h}$ is administered via perfusion, resulting in a significantly more rapid effect (approx. $5 \mathrm{~min}$ ) compared to oral administration. Intravenous therapy should be performed only under observation using a monitoring unit (ICU, IMC-Unit) $[6,7,9]$.

Neostigmine has been used since the 1930s for MG therapy, but no longer plays a role in Germany as an oral medication. However, it is still available and used in Asia, Canada and the United States. A single oral dose is generally $15 \mathrm{mg}$; it is clinically effective after about 15 to $30 \mathrm{~min}$. The effect starts after approx. $5 \mathrm{~min}$ and achieves its maximum 2 to $3 \mathrm{~h}$ later. Parenteral administration is likewise possible (IV, IM, nasal spray).Compared to an oral dose of neostigmine, IV dosage should be reduced by a factor of 30 . Compared to an oral dose of pyridostigmine, dosage should be reduced by a factor of 120 . Parenteral intravenous therapy should be performed only under observation using a monitoring unit (ICU, IMC-Unit); in general 0.15$0.3 \mathrm{mg} / \mathrm{h}$ is administered via perfusion $[6,7,9]$.

For patients who cannot tolerate bromide, ambenonium chloride may be used as an AchE inhibitor. It has been approved in France for MG therapy and can be obtained from an international pharmacy. In Germany ambenonium chloride is not approved, thus reimbursement has to be clarified before use (off-label use). Ambenonium chloride can be administered only orally. A single dose is usually 7.5 to $10 \mathrm{mg}$ ( 5 to $25 \mathrm{mg}$, daily maximum $200 \mathrm{mg}$ ). The effect starts approximately after 20 to $60 \mathrm{~min}$, and the maximum effect can be expected after 6 to $8 \mathrm{~h}$. Since muscarinic side effects occur less frequently than with pyridostigmine bromide ambenonium chloride represents an alternative treatment for patients with MuSK-positive myasthenia gravis, however, more frequent adverse CNS effects have to considered $[6,7,9]$.

Distigmine bromide (Ubretid ${ }^{\circledR}$ ) is only rarely used as an AchE inhibitor for the treatment of MG. A single dose of $20 \mathrm{mg}$ is given orally and the effect starts after 40 to $60 \mathrm{~min}$. Parenteral administration (IV, IM) is likewise possible, but just as with neostigmine and pyridostigmine, a dosage reduction is necessary $(0.5 \mathrm{mg})$. Distigmine bromide degrades very slowly (maximum effect after 12 to $24 \mathrm{~h}$ ); therefore a high risk of accumulation and cholinergic crises has to be considered $[6,7,9]$.

Edrophonium is an intravenous, rapid-acting and short-acting AchEl used for diagnostic purposes only; it reaches its maximum effect after about 1-2 min and rapidly degrades again (so-called edrophonium test, formerly known by its trade name Tensilon ${ }^{\circledR}$ test). Additional administration under ongoing therapy with another AchEl for the purpose of diagnosing a possible oral overdose or underdose is contraindicated, as life-threatening asystole may occur $[6,7,9]$.

\section{Common AchE Inhibitor Side Effects}

Nicotinergic and muscarinergic side effects may occur if dosage is too high (great individual differences) (Tab. 1). Atropine is used as an antidote in a cholinergic crisis [5, 9]. Increased sensitivity to side effects by MuSK-positive MG patients undergoing AchE inhibitor therapy has been described. On the other hand, these patients sometimes require higher dosages to improve their myasthenic symptoms due to poorer response to AchE inhibitors. Special attention should be paid to such patients with respect to the appearance of side effects [9]. 


\section{Immunologically-based Therapy}

Immunological therapy of myasthenia gravis includes both rapidly effective therapies to induce remission as well as long-lasting measures to sustain remission. Rapid improvement within a few days is achieved via apheresis procedures (plasma separation, immunoadsorption) as well as high-dose IV immunoglobulin therapy which is mainly used in the treatment of myasthenic crisis. At the other end of the spectrum, there are markedly delayed immunosuppressive agents that act within months, such as azathioprine and mycophenolate mofetil, as well as thymectomy [10], which are classified as immunological maintenance therapies. Glucocorticosteroids, which induce remission within days to a few weeks are placed between rapid and slow acting treatment regimens. Consideration of the specific "time windows" regarding begin and duration of effectiveness of the immunological therapies is of great importance for individualized treatment, which is usually based on an atleast temporary combination of different immune therapies.

\section{Glucocorticosteroids}

\section{Action mechanism}

Steroids have a broad anti-inflammatory and immunosuppressive action profile. However, the exact mechanisms of action in myasthenia gravis are poorly understood but is likely to include effects on the activation and proliferation of $\mathrm{T}$ and $\mathrm{B}$ lymphocytes as well as the activity of antigen-presenting cells.

\section{Therapeutic value in myasthenia gravis}

Although there is limited evidence from controlled studies, glucocorticosteroids are widely used in all stages and forms of disease when patients do not adequately respond to purely symptomatic treatment with AchEI $[11,12]$. This includes both the purely ocular forms and various degrees of severity of generalized MG up to myasthenic crisis. The use of steroids - assuming sufficient disease severity - is also independent of the classification to a defined subgroup, i. e., both early and late onset, AChR and MuSK antibody-positive, seronegative and thymoma-associated myasthenia gravis are at least temporarily treated with steroids.

\section{Approval and reimbursement}

Prednisone, prednisolone and methylprednisolone are used. Although the latter is not expressly approved for MG treatment, the costs are frequently reimbursed.

\section{Onset of action and dosage}

Based on retrospective studies, the onset of steroid effect in most patients is expected within days to a few weeks, with an overall high response rate of approximately $80 \%[11,12]$. However, a paradoxical deterioration may occur within the first few days, which appears to be more frequent with a high initial dose. Therefore, most centers initially administer a low-dose $10 \mathrm{mg}$ prednisolone equivalent per day as a one-time morning dose in the first week in cases of severe, especially bulbar symptoms, even during initial inpatient monitoring. Following this, a slow dose increase of $5 \mathrm{mg}$ prednisolone equivalent per week can be pursued until stable remission is achieved [13]. However, this strategy is associated with longer la- tency until a clinically satisfactory effect is reached. In many cases, a higher dose of $40-80 \mathrm{mg}$ of prednisolone equivalent per day is administered after the first week $(0.5-1.5 \mathrm{mg} / \mathrm{kg}$ body weight). High-dose pulse therapies (250 to $1000 \mathrm{mg} / \mathrm{d}$ ), as are common in multiple sclerosis, however, are usually obsolete because of an increased risk of initial deterioration. An exception may be ongoing intensive care in the case of myasthenic crisis treated in parallel by apheresis or intravenous immunoglobulins.

\section{Therapy termination or dose reduction}

Once stable remission is achieved, the steroid dose is gradually reduced, e. $\mathrm{g}$. by 5-10 mg every 3-4 weeks, depending on the individual severity and disease progression. Abrupt cessation or rapid reduction should be avoided, as this regularly leads to premature recurrence. Due to the potential long-term adverse effects, the Cushing threshold $(7.5 \mathrm{mg} / \mathrm{d})$ should be achieved within 3 months, when possible.

\section{Contraindications}

According to the approval, only the (rare) case of proven hypersensitivity poses an absolute contraindication for glucocorticosteroid therapy. Therapeutic limitations due to an increased risk of steroid-typical side effects arise in patients with comorbidities such as diabetes mellitus, osteoporosis, infectious diseases, ophthalmological diseases (glaucoma, corneal ulcerations) and psychosis. In these situations, a careful benefit / risk analysis is required to balance the need for effective myasthenia therapy and exacerbation of comorbidity with corresponding, potentially serious complications.

Side effects (see $>$ Table 1)

\section{Laboratory monitoring and other preventive measures}

Close monitoring of blood glucose levels is required especially in high-risk patients and pre-existing diabetes. In addition, if a longer treatment is necessary, osteoporosis prophylaxis and drug-based gastric protection with proton pump inhibitors are advisable.

\section{Azathioprine}

\section{Action mechanism}

Azathioprine (AZT) is a purine analogon converted into active and inactive metabolites by various enzymatic systems; the formation of mercaptopurine by glutathione-S-transferase is of particular importance. As an atypical nucleoside, mercaptopurine interferes with DNA and RNA synthesis, thus leading to antiproliferative and proapoptotic effects on $T$ and $B$ lymphocytes resulting in lymphopenia. AZT and its active metabolites are degraded to uric acid via xanthine oxidase and methylated by the thiopurine S-methyltransferase (TPMT) and thereby inactivated.

\section{Therapeutic value in myasthenia gravis}

AZT is the drug of first choice for MG immunosuppressive maintenance therapy. Its long-term stabilizing effect on disease progression has been proven by a randomized controlled trial that demonstrated a lower steroid demand and sustained decline in AChR Ab titers in AZT-treated patients, but only beyond the first year of treat- 
- Table 1 Relevant adverse effects of therapy.

\section{Medication}

Achetylcholinesterase inhibitors (AchEI)

Glucocorticosteroids

Azathioprine (AZT)

Mycophenolate mofetil (MMF)

Methotrexate (MTX)

Cyclosporin A (CSA)

Tacrolimus (TAC)

Intravenous immunoglobulin

\section{Side effects}

Nicotinergic: fasciculation, muscle cramps, muscle weakness Muscarinergic: gastrointestinal: diarrhea, nausea, vomiting, abdominal cramps; respiratory: increased bronchial secretion, bronchoconstriction; cardiovascular: bradycardia, arterial hypertension, intermittent sinus tachycardia; ophthalmological: poor accommodation with blurred vision, miosis; secretory: hypersalivation, increased sweating, tears; central nervous: anxiety, agitation, disorientation, sleeplessness, headaches, epileptic seizures, loss of consciousness

latrogenic Cushing's syndrome, weight gain, obesity, steroid acne, steroid diabetes, susceptibility to infection, thrombosis, increase in blood pressure, hypokalemia, edema; especially with long-term therapy: osteoporosis, aseptic bone necrosis, cataracts, glaucoma, gastric and duodenal ulcers, steroid myopathy, sleep disorders, psychiatric side effects (psychosis)

hepatotoxicity, myelosuppression (leuco-, thrombocytopenia, megaloblastic anemia) - Caution comedication with allopurinol, gastrointestinal (diarrhea, nausea, vomiting), immediate idiosyncratic reaction, arthralgia, myalgia, pancreatitis, cholestasis, alveolitis, opportunistic infections, skin eruption, increased risk of malignancy with increasing duration of therapy and increasing cumulative dose

Gastrointestinal (diarrhea, nausea, vomiting, abdominal pain), edema, myelodepression (leukopenia, erythroblastopenia, hemolytic anemia), infections (including opportunistic infections such as progressive multifocal leucencephalopathy, candidiasis, Caution hepatitis reactivation), increased risk of malignancy especially of the skin (Caution protection in the sun and UV light exposure)

Gastrointestinal (nausea, vomiting, abdominal pain), myelodepression (leuko-thrombocytopenia), stomatitis, anorexia, reduction of creatinine clearance, arthralgia, myalgia, alopecia, osteoporosis, retroperitoneal and pulmonary fibrosis, mucositis, alveolitis, pneumonitis, increased risk of malignancy

Nephrotoxicity, hypertension, cholestasis, gastrointestinal (nausea, vomiting, diarrhea), gingival hyperplasia, hypertrichosis, headache, rashes, acne, thrombocytopenia, anemia, allergic reactions, seizures, dysmenorrhoea, encephalopathy, myopathy, leukopenia, pancreatitis, hyperthermia, increased malignancy risk (especially lymphoproliferative neoplasia), tremor

Nephrotoxicity, hypertension, cholangitis / hepatitis, neurotoxicity (dose-dependent), gastrointestinal (nausea, vomiting, diarrhea, constipation), arthralgias, anemia, thrombocytopenia, rashes, myopathy, cardiomyopathy, pancreatitis, allergic / anaphylactic reactions, vasculitis, increased risk of malignancy (especially lymphoproliferative neoplasia)

Chills, headache, dizziness, fever, vomiting, allergic reactions, nausea, joint pain, hypotension, lumbar pain, anaphylactic reactions (Caution IgA deficiency), hemolytic anemia, myocarditis, arthritis, acute renal failure, congestive heart failure, arterial hypertension, tachycardia, thromboembolism aseptic meningitis, encephalopathy ment [14]. AZT is primarily indicated with relevant severity of symptoms or in the case of long-term or repeated need for steroid treatment. Patients with late-onset AChR Ab-positive myasthenia gravis are often treated primarily with combined steroid and AZT immunosuppression if the disease severity is high. In contrast, in early-onset AChR Ab-positive myasthenia the effect of thymectomy may be awaited when gradually reducing steroid therapy $[10,15]$. AZT may be started with relapses. For MuSK Ab-positive MG, the B cell-depleting monoclonal antibody rituximab may be more effective than the AZT [16], and is thus frequently the first choice immunotherapy for severely-affected MuSK Ab-positive patients.

\section{Approval and reimbursement}

AZT is an approved immunosuppressant for the treatment of myasthenia gravis.

\section{Dosage}

The standard dosage of AZT is $2-3 \mathrm{mg} / \mathrm{kg}$ body weight/day. In the case of genetic TPMT hypoactivity or comedication with the xanthine oxidase inhibitor allopurinol, a reduction to $25 \%$ of the standard dose must be made in order to prevent severe myelotoxicity.
In any case, the administration of a single test dose of $50 \mathrm{mg}$ is recommended prior to administration of the full dose. There is an absolute contraindication to the therapy if this leads to acute intolerance with occurrence of fever, myalgia, vomiting and fever (idiosyncratic immediate reaction, <1\%) [17].

\section{Onset of effect}

The onset of the effect of AZT is significantly delayed compared to steroid therapy. A relevant effect on disease activity is expected at the earliest after 6 months, but usually after 12 months treatment duration. Thus, AZT therapy is usually combined with overlapping steroid treatment.

\section{Therapy termination or dose reduction}

Once stable remission has been achieved, and steroid medication is gradually reduced as far as possible, AZT is usually continued for at least 5 years. Following this, slow cessation of azathioprine in 25-50 mg increments every 3 to 12 months may be considered. However, even after prolonged periods of therapy and stable remission, there should be no abrupt withdrawal, as this can lead to severe relapses of the disease [18]. Close clinical monitoring is in- 
dispensable during a gradual reduction of medication. Laboratory controls exhibiting increasing auto-antibody titers can provide early indication of exacerbation of the disease due to dose reduction and therefore can also be helpful for monitoring therapy.

\section{Contraindications}

Pre-existing severe liver and bone marrow damage as well as severe renal insufficiency are contraindications.

\section{Side effects (see $>$ Table $\mathbf{1}$ ).}

Larger studies demonstrated increased risk of malignancies (lymphoma, non-melanoma-related skin cancer) as a result of increased duration of therapy and increasing cumulative AZT dose $[19,20]$.

\section{Laboratory controls}

After starting AZT treatment every 1 to 2 weeks monitoring of white blood count (WTC), liver and kidney values as well as amylase levels are recommended. In the longer term, 4 to 6-week, control intervals of 3-6 months are sufficient. As a rule, relative and absolute lymphopenia as well as an increase of MCV are observed, which can also be regarded as an indicator of effective therapy. On several occasions, a therapeutic range of absolute lymphocyte count between 600 and $1000 / \mu$ l with total leukocyte numbers $>3500 / \mu \mathrm{l}$ is attempted, but this is not handled uniformly. Additionally, a steroid induced leucocytosis has to be considered. Liver enzyme elevations are relatively frequently observed and may, if sustained, require cessation of AZT treatment and a change to a second-choice immunosuppressant.

\section{Mycophenolate Mofetil}

\section{Mechansim of action}

Mycophenolate mofetil (MMF) is metabolized in the body into the active substance mycophenolic acid. Mycophenolic acid selectively and reversibly inhibits the enzyme inosine monophosphate dehydrogenase, resulting in a reduction of purine synthesis and reduced proliferation of $\mathrm{T}$ and $\mathrm{B}$ lymphocytes.

\section{Therapeutic value in myasthenia gravis}

Due to its relatively favorable risk / benefit profile, MMF now represents the first alternative in the case of intolerable side effects or inefficacy of AZT. In contrast to clinical experience, two randomized, controlled, prospective studies failed to demonstrate significant benefit of MMF. However, these negative results are not considered meaningful. In one study, the observation period of 3 months (2.5 g MMF/day combined with $20 \mathrm{mg}$ prednisone/day) compared to placebo combined with $20 \mathrm{mg}$ prednisone/day was clearly too short [21]. In a second study, $2 \mathrm{~g} \mathrm{MMF/day} \mathrm{was} \mathrm{tested}$ versus placebo over nine months. Both groups additionally received prednisone, so that the steroid-saving effect was evaluated. Criticisms of this study likewise included the too-short observation period of nine months, the insufficient MMF daily dose and that the placebo arm reflected a higher than expected therapeutic effect of prednisone [22]. In an open-ended long-term therapy study of ACh receptor $A b$-positive patients, a steroid-sparing effect of MMF with a median daily dose of $2 \mathrm{~g} /$ day ( $500 \mathrm{mg}$ to $3 \mathrm{~g} /$ day) was only detec- ted after six months, but became increasingly apparent with continued therapy [23].

\section{Approval and reimbursement}

In Germany an off-label indication for MMF exists since 2013; reimbursement has been based on the following prerequisites: non-tolerance of AZT or insufficient response to the drug, so that steroid dose cannot be lowered under the Cushing threshold (publication of the GBA-German national medico-economic board, 2014). As a note of caution, the clinical data for myasthenia gravis and limited approval relate only to MMF (prodrug of mycophenolic acid), and not to mycophenolic acid which is commercially available as mycophenolate sodium (Myfortic ${ }^{\circledR}$, produced by Novartis). 720 mg of mycophenolate sodium corresponds to $1 \mathrm{~g}$ mycophenolate mofetil.

\section{Dosage}

The optimal MMF dosage has yet to be systematically studied. The usual dose ranges between 1-3 g/day [24]. Based on previous controlled therapy studies, at the start of treatment a target dose of 2 to $2.5 \mathrm{~g} /$ day should desired divided into two single doses $(2 \times 1 \mathrm{~g}$ or $2 \times 1.25 \mathrm{~g} /$ day). In the event of abdominal complaints, further division into several single doses and/or a temporary dose reduction and gradual dosage increase is recommended. After remission and reaching stable disease, a slow dose reduction may be attempted after several years (e. g., five years) (see below) [25].

In transplantation medicine the target serum level is $1-2 \mathrm{mg} / \mathrm{I}$ when determined $12 \mathrm{~h}$ after the last dose (trough level). In MG, MMF dosage is usually not directed according to serum levels (drug monitoring) but rather according to clinical effect.

\section{Changing from AZT or cyclosporin to MMF and interactions}

Combining MMF and AZT is pharmacologically not useful, since both substances result in a reduction of nucleic acid synthesis which causes additive toxicity. When switching from AZT to MMF, a fourweek treatment break should be maintained after completion of AZT treatment to avoid excessive immunosuppression. Unlike AZT, there is no interaction between MMF and allopurinol.

In transplantation medicine, MMF is sometimes combined with cyclosporin A. However, there are no meaningful data regarding myasthenia gravis. However, when switching cyclosporin to MMF, it is recommended to overlap the therapy, that is, cyclosporin therapy should be continued for several months [26].

\section{Onset of effect}

In general, with MMF the treatment effect is observed somewhat more earlier than under AZT, but can also take 6 to 12 months.

\section{Therapy termination or dose reduction}

A too-rapid dose reduction is related to an increased risk of recurrence [27]; therefore a slow reduction by $500 \mathrm{mg} /$ day every 12 months is recommended [25].

\section{Contraindications}

MMF cannot be used if there is a known hypersensitivity to the drug as well as during pregnancy and lactation.

Side effects (see $>$ Table 1 ) 


\section{Laboratory controls}

In the first month, there should be a weekly monitoring of WTC, liver enzymes, creatinine. In the 2 nd and 3rd month controls every 2 weeks and from the 4 th month onward once a month are recommended.

\section{Methotrexate}

\section{Mechanism of action}

Methotrexate (MTX) is a folic acid antagonist that competitively inhibits the enzyme dihydrolate reductase and thus the formation of tetrahydrofolic acid. Tetrahydrofolic acid deficiency leads to a reduced synthesis of thymidine and purine bases and thus results in a reduced formation of DNA and RNA with the consequence of impaired cell function and apoptosis. MTX also inhibits the proliferation of activated $T$ and $B$ lymphocytes as well as restricts the number of antibody-producing plasma cells.

\section{Therapeutic value in myasthenia gravis}

MTX represents an alternative to AZT in case of intolerance or inadequate effect of that drug. For elderly patients, administering MTX once a week can improve compliance. MTX is the drug of choice in the case of accompanying rheumatoid arthritis.

There is one 24-month randomized, single blinded study comparing MTX in an oral dose of $17.5 \mathrm{mg} /$ week plus prednisone to AZT (2.5-3 mg/kg body weight/day) plus prednisone [28]. This study demonstrated a cortisone-sparing effect comparable to AZT. In contrast, a placebo-controlled trial using an oral dose of $20 \mathrm{mg} \mathrm{MTX/}$ week showed no prednisone-sparing effect within 12 months compared to placebo [29]. However, the study included only 25 patients in each treatment arm, thus the too small cohort and the too short duration allow no further conclusions regarding effectiveness.

\section{Approval}

MTX is not approved for the treatment of myasthenia gravis.

\section{Dosage}

15-20 mg per week can be administered subcutaneously or orally. Parenteral administration is related to greater bioavailability [30]. In a study by Heckmann et al. [28], the initial dose was $7.5 \mathrm{mg} /$ week and was increased by $2.5 \mathrm{mg} /$ week until $17.5 \mathrm{mg} /$ week was reached. At the same time, in order to improve the tolerability of MTX, a folic acid substitute must be established, usually at a dose of $10 \mathrm{mg} /$ week orally on the day after each MTX administration.

\section{Changing from AZT to MTX and interactions}

Combining MTX and AZT is pharmacologically not useful, since both substances result in a reduction of nucleic acid synthesis which causes additive toxicity. When switching from AZT or MTX to MMF, a four-week treatment break should be taken after completion of AZT or MTX treatment to avoid excessive immunosuppression. Likewise, a combination of cyclosporin and MTX is not appropriate due to the risk of hyperimmunosuppression.

Drugs that show increased plasma protein binding or NSAIDs can lead to increased toxicity of MTX. Sufficient hydration should be ensured, especially in elderly patients.

\section{Onset of effect}

According to Heckmann's data [28], the steroid sparing effect began after about 10 months and compared to AZT was somewhat earlier.

\section{Therapy termination or dose reduction}

There are no systematic investigations regarding ending therapy or reducing MTX dosage. Similar to other substances (AZT, MMF), it can be assumed that a slow decrease of MTX is also advisable to reduce the risk of recurrence. Likewise, this should be considered only after several years of stable remission.

\section{Contraindications}

MTX must not be used in cases of known hypersensitivity to the drug, renal impairment with creatinine clearance $<60 \mathrm{ml} / \mathrm{min}$, severe hepatic impairment, hematopoietic dysfunction, increased alcohol consumption, immunodeficiency, active infections, stomatitis, gastrointestinal ulcers, pregnancy and lactation.

\section{Side effects $($ see $>$ Table 1$)$}

\section{Special situations}

Dosage should be temporarily reduced in case of aphthous ulcers. In the case of myelosuppression with leukocyte counts $<3000 / \mu \mathrm{l}$, MTX should be paused, followed by treatment with a reduced dosage. MTX must be discontinued if liver enzymes increase more than 3 times the upper threshold. If creatinine clearance decreases, the MTX dose should be adjusted according to the prescribing information.

\section{Controls and monitoring}

Data relating to the following should be available prior to initiating therapy: WTC, liver enzymes, bilirubin, serum albumin, renal values (with creatinine clearance), hepatitis serology; if necessary; tuberculosis should be ruled out. For the first few weeks, monitoring WTC should be performed weekly, then biweekly. Monthly controls should include inspection of the oral cavity and pharynx for mucosal changes (aphthous ulcers, thrush), WTC, liver enzymes, creatinine, queries regarding pulmonary dysfunction.

\section{Cyclosporin A}

\section{Mechanism of action}

Cyclosporin A (CSA) inhibits the transcription of cytokines such as interleukin-2 and interferon-gamma via binding to calcineurin, thereby inhibiting the activation of T lymphocytes.

\section{Therapeutic value in myasthenia gravis}

Due to its spectrum of side effects, treatment requires an intensive monitoring. Due to available drug alternatives, CSA is a reserve drug, and therefore rarely administered in Germany.

\section{Approval}

CSA is not approved for the treatment of myasthenia gravis.

\section{Dosage}

The usual initial dose is $3-4 \mathrm{mg} / \mathrm{kg}$ body weight with a subsequent reduction to $2-3 \mathrm{mg} / \mathrm{kg}$ body weight, divided between two daily 
doses [31], This recommended dosage is lower than in a placebocontrolled study using $6 \mathrm{mg} / \mathrm{kg}$ body weight [32]. The CSA target level (trough level prior to morning intake) is $<200 \mathrm{ng} / \mathrm{ml}$ and about $100 \mathrm{ng} / \mathrm{ml}$ during maintenance therapy [31]. These dosages and serum levels are for orientation purposes only. The dosage must be based on the clinical findings, renal values and blood pressure.

\section{Interactions}

Displacement from plasma protein binding leads to numerous interactions with other drugs, including increase of the CSA serum level by macrolide antibiotics, calcium antagonists, narcotics, steroids, or decrease in CSA levels by carbamazepine, barbiturates, phenytoin, metamizole, rifampicin, St. John's wort (refer to prescribing information).

\section{Onset of effect}

CSA can take noticeable effect after 1-2 months and reaches its maximum effect after a median of 7 months [33].

\section{Therapy termination or dose reduction}

If creatinine increases by more than $50 \%$ or $>1.5 \mathrm{mg} \%$, the dose must be reduced or treatment discontinued.

\section{Contraindications}

These include known hypersensitivity to CSA, renal insufficiency, uncontrolled high blood pressure, uncontrolled infectious diseases, history of known or diagnosed malignant tumors.

\section{Side effects (see $>$ Table 1 )}

Controls and monitoring In the initial adjustment phase: CSA level (trough level prior to morning dose): 2x/week, plus 1x/week WTC, liver enzymes, creatinine followed by monthly controls.

\section{Tacrolimus}

\section{Mechanism of action}

Tacrolimus (TAC) has an action mechanism similar to CSA and interacts with the inhibition of calcineurin with calcium-dependent signal transduction during $\mathrm{T}$ lymphocyte activation.

\section{Therapeutic value in myasthenia gravis}

Unlike in Asian countries (approved in Japan), the significance of TAC is relatively low, due to the limited trials, spectrum of side effects, high monitoring requirements and the availability of drug alternatives. To date there are no sufficiently long-running randomized, double-blind, placebo-controlled trials in myasthenia gravis.

\section{Approval}

TAC is not approved by the EMEA for the treatment of myasthenia gravis.

\section{Dosage}

TAC is administered at a dosage of $0.1 \mathrm{mg} / \mathrm{kg}$ body weight, divided in two individual doses [34]. The TAC target level (trough level prior to morning intake) is $7-8 \mathrm{ng} / \mathrm{ml}$ [35]. These dosages are for orientation purposes only.

\section{Interactions}

TAC is metabolized in the liver via CYP3A4. There are strong interactions with antimycotics, macrolide antibiotics, HIV protease inhibitors and numerous other medications (see prescribing information). Grapefruit juice can also raise the TAC blood level; likewise there is frequently a rise in the potassium level.

\section{Onset of effect}

TAC can show noticeable effects after $1-2$ months and reaches its maximum effect after a median of 6 months [36].

\section{Therapy termination or dose reduction}

There are no systematic surveys regarding treatment ending or reducing TAC dosage.

\section{Contraindications}

TAC may not be used if there is known hypersensitivity to the drug or other macrolides.

Side effects $($ see $>$ Table 1$)$

\section{Controls and monitoring}

In the initial adjustment phase: TAC level (trough level prior to morning dose): 2x/week, plus 1x/week WTC, liver enzymes, creatinine, electrolytes followed by monthly controls.

\section{Intravenous Immunoglobulins (IVIG)}

\section{Mechanism of action}

Intravenous immunoglobulins are made from pooled plasma from at least 1000 donors. The mechanism of action of intravenous immunoglobulins (IVIG) is not yet fully understood. Important factors include neutralization of circulating autoantibodies, down-regulation of pathological B cell clones, blockade of Fc receptors, influence on $T$ cell function, cytokine interactions and reduced complement activation.

\section{Therapeutic value in myasthenia gravis}

The use of IVIG is an established treatment option in the case of myasthenic crisis and crisis-related exacerbation of myasthenic symptoms. Since 2014 the GBA has approved IVIG for off-label use which guarantees reimbursement in the outpatient sector in the event of a "myasthenic crisis", or "severe exacerbation" of myasthenic symptoms in order to avoid critical deterioration. Refer to a separate article in this special issue for the definition of both clinical conditions. Careful documentation of the clinical findings is important. Long-term interval therapy with IVIG is not permitted. If this appears to be necessary after exhausting other alternative therapies, an application for reimbursement must be made as part of an individual therapeutic process. There are no controlled studies of long-term therapy, but several case series demonstrate its efficacy in treatment of otherwise refractory patients or if there are unacceptable side effects in alternative therapies [37-39]. 


\section{Dosage}

Several IVIG preparations from different manufacturers are available, but without any indication of effects or tolerability differences. According to the GBA approval, IVIG preparations by the following manufacturers may be prescribed for off-label use: Axicorp, Baxter, Biotest, Behring, Grifols, Kedrion, Octapharma. In case of a myasthenic crisis or severe exacerbation, intravenous administration of $0.4 \mathrm{~g} / \mathrm{kg}$ body weight is given daily for 5 consecutive days or $1 \mathrm{~g} / \mathrm{kg}$ divided among 2-3 days. IVIG is also an option in special situations, e. g. to bridge the onset of immunosuppressive long-term therapy when faced with prednisolone adverse effects or contraindications. After proof of efficacy applying $0.4 \mathrm{~g} / \mathrm{kg} / \mathrm{d}$ over 5 days, further single administration of $0.4 \mathrm{~g} / \mathrm{kg} / \mathrm{d}$ may be used at individually-determined intervals (generally between 4 and 12 weeks) in anticipation of deterioration of myasthenic symptoms.

\section{Onset of effect}

Latency of IVIG efficacy is a few days.

\section{Therapy termination or dose reduction}

Therapy can terminate, for example when immunosuppressive long-term therapy becomes effective, by extension of therapy intervals or reducing treatment days.

\section{Contraindications}

IVIG must not be used if there are known previous allergic reactions. Likewise, it should not be used in patients with antibodies against IgA and those patients with selective IgA deficiency.

Side effects $($ see $>$ Table 1$)$

\section{Controls and monitoring}

The IgA and serum creatinine levels should be determined prior starting therapy. In elderly patients, IVIG should be administered via slow infusion to avoid overload and hyperviscosity syndrome. The administration of IVIG, in particular the initial dose, must be supervised by a physician so that immediate treatment of allergic reaction is possible.

\section{Individualized Therapy}

The basis of an individualized therapy of myasthenia gravis includes both severity and dynamics of the clinical disease, as well as the immunologically-based classification to a specific subgroup.

The initial therapy for all patients is symptomatic treatment with AChE inhibitors, started immediately after a diagnosis is determined as well as in ambiguous cases with lack of ancillary findings. In such cases AChE inhibitors might be used as an aid to diagnosis. Here, the provisional administration of pyridostigmine often replaces the previously obligatory Tensilon or edrophonium chloride test, especially in an outpatient setting.

If satisfactory improvement cannot be achieved with symptomatic therapy alone, or if severe clinical manifestation is present from the outset, steroid therapy will usually be indispensable. In particular, the presence of bulbar and respiratory symptoms may require initial treatment in the hospital. Additional intervention with apheresis or high-dose immunoglobulin administration with intensive care treatment may be necessary when severe or rapidly progressive symptoms are present.
In addition, the indication for immunosuppressive or immunomodulatory long-term therapy needs to be evaluated, primarily in patients with relevant disease severity and a long-term need for steroid therapy. First and foremost, patients with late-onset AChR Ab-positive generalized myasthenia gravis are treated with a combination of steroids and AZT, as these patients are not suitable for thymectomy due to thymic atrophy and have an increased risk of serious complications in high-dose monotherapy with steroids. In the case of early-onset AChR Ab-positive MG, which is usually associated with thymic hyperplasia, attempts can first be made to gradually eliminate steroid therapy after surgery has been performed and to start immunosuppressants only when relapses occur in the further course of the disease.

Thymoma-associated and MuSK Ab-positive myasthenia gravis have specific characteristics. In these conditions, disease course is often severe and more difficult to treat. Thymoma-associated MG is an absolute indication for thymus surgery, independent of the severity of the myasthenia. Nevertheless, many thymoma patients do not postoperatively improve with respect to myasthenia; thus in many cases combined immunosuppressive treatment has to be used, if necessary with second-choice immunosuppressants or other therapies. MuSK Ab-positive myasthenia gravis frequently does not respond well to the usual symptomatic and immunosuppressive therapies, but frequently shows good improvement under therapy with the B cell-depleting monoclonal antibody rituximab, such that it is frequently used as the first-choice immunotherapy for these patients. Additional articles a special issue of Aktuelle Neurologie provide further details regarding these special forms of myasthenia gravis.

Purely ocular myasthenia represents a special manifestation of MG. In many cases symptomatic treatment with AchEl is sufficient for symptom control. In other patients a combination with steroids is necessary, whereas the use of long-term immunosuppressive therapy is not handled uniformly. Previous data indicate that secondary generalization of ocular myasthenia less often occurs under steroid therapy [40]. The risk of secondary generalization was also used as an argument for the early use of azathioprine. On the other hand, other centers only use AZT when relapses occur after steroid tapering. In the case of purely ocular myasthenia and absence of thymoma, thymectomy is normally cautiously indicated.

Drug treatment of myasthenia gravis in advanced age poses further challenges, as well as additional associated autoimmune diseases and paraneoplastic forms, which due to space requirements have not been discussed here. We therefore refer to more comprehensive monographs $[41,42]$.

Special problems during pregnancy and lactation are considered in another article in the current special issue.

\section{SUMMARY}

In the majority of patients, myasthenic symptoms can be well controlled by the drugs described in this article. Nevertheless, an individual approach is always necessary in consultation with a well-informed patient. Likewise, the disease requires regular monitoring of the clinical effect and side effects, and, if necessary, adaptation of the therapy. 


\section{Conflict of Interest}

The authors declare no conflict of interest.

\section{References}

[1] Gilhus NE, Verschuuren JJ. Myasthenia gravis: Subgroup classification and therapeutic strategies. Lancet Neurol 14: 1023-1036

[2] Aeschlimann JA, Reinert M. Pharmacological action of some analogues of physiostigmine. J Pharmacol Exp Ther 1931; 43: 413-444

[3] Remen L. Zur Pathogenese und Therapie der Myasthenia gravis pseudoparalytica. Dtsch Z Nervenheilkunde 1932; 128: 66-78

[4] Walker MB. Treatment of myasthenia gravis with physostigmine. Lancet 1934; 1: 1200-1201

[5] Nair VP, Hunter JM. Anticholinesterases and anticholinergic drugs. Continuing Education in Anaesthesia, Critical Care \& Pain 2004; 4: 164-168

[6] Maggi L, Mantegazza R. Treatment of myasthenia gravis. Focus on Pyridostigmine. Clin Drug Investig 2011; 31: 691-701

[7] Deutsche Gesellschaft für Neurologie (DGN). Leitlinien der DGN: Diagnostik der Myasthenia gravis und des Lambert-EatonSyndroms.(29.06.2015). Im Internet: http://www.dgn.org/leitlinien/ 3005-II-68-II-diagnostik-und-therapie-der-myasthenia-gravis-unddes-lambert-eaton-syndroms

[8] Mehndiratta MM, Pandey S, Kuntzer T. Acetylcholinesterase inhibitor treatment for myasthenia gravis. Cochrane Database Syst Rev 2014; CD006986 doi:10.1002/14651858.CD006986.pub3

[9] Punga RA, Stalberg E. Acetylcholinesterase inhibitors in myasthenia gravis: To be or not to be? Muscle Nerve 2009; 39: 724-728

[10] Wolfe GI, Kaminski H], Aban IB et al. Randomized trial of thymectomy in myasthenia gravis. N Engl J Med 2016; 375: 511-522

[11] Pascuzzi RM, Coslett HB, Johns TR. Long-term corticosteroid treatment of myasthenia gravis: report of 116 patients. Ann Neurol 1984; 15: 291-298

[12] Schneider-Gold C, Gajdos P, Toyka KV et al. Corticosteroids for myasthenia gravis. Cochrane Database Syst Rev 2005; 2 CD002828

[13] Seybold ME, Drachman DB. Gradually increasing doses of prednisone in myasthenia gravis. Reducing the hazards of treatment. N Engl J Med 1974; 290: 81-84

[14] Palace J, Newsom-Davis J, Lecky B. A randomized double-blind trial of prednisolone alone or with azathioprine in myasthenia gravis. Myasthenia Gravis Study Group. Neurology 1998; 50: 1778-1883

[15] Gronseth GS, Barohn R]. Practice parameter: Thymectomy for autoimmune myasthenia gravis (an evidence-based review): report of the Quality Standards Subcommittee of the American Academy of Neurology. Neurology 2000; 55: 7-15

[16] Iorio R, Damato V, Alboini PE et al. Efficacy and safety of rituximab for myasthenia gravis: A systematic review and meta-analysis. J Neurol 2015; 262: 1115-1119

[17] Hohlfeld R, Michels $M$, Heininger $K$ et al. Azathioprine toxicity during long-term immunosuppression of generalized myasthenia gravis. Neurology 1988; 38: 258-261

[18] Hohlfeld R, Toyka KV, Besinger UA et al. Myasthenia gravis: reactivation of clinical disease and of autoimmune factors after discontinuation of long-term azathioprine. Ann Neurol 1985; 17: 238-242

[19] Pedersen EG, Pottegard A, Hallas J et al. Myasthenia and risk of cancer: A population-based case-control study. Eur J Neurol 2014; 21: 773-778

[20] Pedersen EG, Pottegard A, Hallas J et al. Risk of non-melanoma skin cancer in myasthenic patients treated with azathioprine. Eur J Neurol 2014; $21: 454-455$
[21] Muscle Study Group. A trial of mycophenolate mofetil with prednisone as initial immunotherapy in myasthenia gravis. Neurology 2008; 71 : 394-399

[22] Sanders DB, Hart IK, Mantegazza R et al. An international, phase III, randomized trial of mycophenolate mofetil in myasthenia gravis. Neurology 2008; 71: 400-406

[23] Hehir MK, Burns TM, Alpers J et al. Mycophenolate mofetil in AchR-antibody-positive myasthenia gravis: outcomes in 102 patients. Muscle Nerve 2010; 41: 593-598

[24] Meriggioli MN, Ciafaloni E, Al-Hayk KA et al. Mycophenolate mofetil for myasthenia gravis. Neurology 2003; 61: 1438-1440

[25] Hobbson-Webb LD, Hehir M, Crum B et al. Can mycophenolate mofetil be tapered safely in myasthenia gravis? A retrospective, multicenter analysis. Muscle Nerve 2015; 52: 211-215

[26] Lim AKH, Donnan G, Chambers B et al Mycophenolate mofetil substitution for cyclosporine-dependent myasthenia gravis and nephrotoxicity. Intern Med J 2007; 37: 55-59

[27] Oskarsson B, Rocke DM, Dengel K et al. Myasthenia gravis exacerbation after discontinuing mycophenolate. Neurology 2016; 86: 1-5

[28] Heckmann JM, Rawoot A, Bateman K et al. A single-blinded trial of methotrexate versus azathioprine as steroid-sparing agents in generalized myasthenia gravis. BMC Neurology 2011; 11: 97

[29] Pasnoor M, He J, Herbelin L et al. A randomized controlled trial of methotrexate for patients with generalized myasthenia gravis. Neurology 2016; 87: 57-64

[30] Braun J, Kaestner P, Flaxenberg P et al. MC-MTX.6/RH Study Group. Comparison of the clinical efficacy and safety of sucutaneous versus oral administration of methotrexate patients with active rheumatoid arthritis. Arthritis Rheum 2008; 58: 73-81

[31] Nagane Y, Suzuki S, Suzuki N et al. Two-year treatment with cyclosporine microemulsion for responder myasthenia gravis patients. Eur Neurol 2010; 64: 186-190

[32] Tindall RSA, Rollins JA, Phillips JT et al. Preliminary results of a double blind, randomized, placebo-controlled trial of cyclosporine in myasthenia gravis. N Engl ] Med 1987; 316: 719-724

[33] Ciafoloni E, Nikhar NK, Massey JM et al. Retrospective analysis of the use of cyclosporine in myasthenia gravis. Neurology 2000; 55: 448-450

[34] Ponseti JM, Gamez J, Azem J et al. Tacrolimus for myasthenia gravis: A clinical study of 212 patients. Ann NY Acad Sci 2008; 1132: 254-263

[35] Cruz JL, Wolff ML, Vanderman AJ et al. The emerging role of tacrolimus in myasthenia gravis. Ther Adv Neurol Disord 2015; 8: 92-103

[36] Nagaishi A, Yukitake M, Kuroda Y. Long-term treatment of steroiddependent myasthenia gravis patients with low-dose tacrolimus. Inter Med 2008; 47: 731-736

[37] Eienbröker C, Seitz F, Spengler A et al. Intravenous immunoglobulin maintainance treatment in myasthenia gravis: A randomized, controlled trial sample size simulation. Muscle Nerve 2014; 50: 999-1004

[38] Liew WKM, Powell CA, Sloan SR et al. Comparison of plasmapheresis and intravenous immunoglobulin as maintenance therapies for juvenile myasthenia gravis. JAMA Neurol 2014; 71: 575-580

[39] Zinman L, NG E, Bril V et al. IV immunoglobulin in patients with myasthenia gravis: a randomized controlled trial. Neurology 2007; 68: 837-841

[40] Kupersmith M], Latkany R, Homel P. Development of generalized disease at 2 years in patients with ocular myasthenia gravis. Arch Neurol 2003; 60: 243-248

[41] Köhler W, Sieb JP, Hrsg. Myasthenia gravis. Bremen: Uni-Med Verlag; 2012

[42] Engel AG, ed. Myasthenia gravis and Myasthenic Disorders. Oxford: Oxford University Press; 2012 